\title{
Changes in parotid gland morphology and function in patients treated with intensity-modulated radiotherapy for nasopharyngeal and oropharyngeal tumors
}

\author{
Kenichi Obinata - Motoyasu Nakamura • \\ Marco Carrozzo • Iain Macleod • Andrew Carr • \\ Shinichi Shirai • Hitoshi Ito
}

Received: 19 March 2013/Accepted: 30 July 2013/Published online: 25 August 2013

(C) The Author(s) 2013. This article is published with open access at Springerlink.com

\begin{abstract}
Objective To evaluate the morphological changes of the parotid glands in patients treated with intensity-modulated radiotherapy (IMRT) for nasopharyngeal and oropharyngeal tumors and the correlations with parotid function.

Methods Ten patients with nasopharyngeal and oropharyngeal tumors treated with IMRT between May 2009 and January 2010 at Hokkaido University Hospital were included in this study. In the morphological assessment of the parotid glands, the sizes and computed tomography (CT) numbers of the bilateral parotid glands before and after IMRT with CT were calculated. For functional assessment of the parotid glands, we conducted the Saxon test and used a visual analog scale (VAS) for xerostomia evaluation.

Results Reductions in saliva secretion were observed in the patients treated with IMRT for nasopharyngeal and oropharyngeal tumors, and there was a significant
\end{abstract}

\footnotetext{
K. Obinata $(\bowtie)$

Department of Dental Radiology, Center for Dental Clinics,

Hokkaido University Hospital, Kita-13, Nishi-7, Kita-ku,

Sapporo, Hokkaido 060-8586, Japan

e-mail: obikenca@yahoo.co.jp

M. Nakamura

Nakamura Dental Clinic, Sapporo, Japan

M. Carrozzo

Department of Oral Medicine, University of Newcastle upon

Tyne, Newcastle upon Tyne, UK

I. Macleod · A. Carr

Department of Dental Radiology, University of Newcastle upon Tyne, Newcastle upon Tyne, UK

S. Shirai $\cdot$ H. Ito

Omni Dentix, Sapporo, Japan
}

correlation between the reduction in saliva secretion and the VAS. The reductions in the parotid gland size and CT number were larger on the ipsilateral side than on the contralateral side. The reduction in saliva secretion was not significantly correlated with the reduction in parotid gland size, but was significantly correlated with the reduction in CT number.

Conclusions Morphological and functional changes of the parotid glands were observed after IMRT for nasopharyngeal and oropharyngeal tumors, and preservation of the contralateral parotid glands was only partly achieved. Among the morphological changes of the parotid glands, the CT number may be considered a predictor of parotid function after radiotherapy.

Keywords Intensity-modulated radiotherapy (IMRT) · Parotid gland · Nasopharyngeal and oropharyngeal tumors $\cdot$ Morphological and functional changes

\section{Introduction}

Radiotherapy plays an important role in head and neck tumor treatment because of the cosmetic and functional preservation that becomes possible. Despite advances in radiotherapy equipment and irradiation technology, morbidity related to radiotherapy is not completely avoidable [1]. Xerostomia is a common adverse effect of radiotherapy for head and neck tumors, and results in reduced patient quality of life by inducing dysphagia, dysgeusia, caries, and periodontitis [2]. In recent years, our hospital has used intensity-modulated radiotherapy (IMRT) for patients with head and neck tumors to enable preservation of the major salivary glands. It has been reported that 
IMRT is superior to conventional radiotherapy in regard to dose, dosimetry, and preservation of salivary gland functions [3, 4]. However, we have experienced a variety of subjective complaints of xerostomia in patients treated with IMRT. The present study aimed to evaluate the morphological changes of the parotid glands in patients treated with IMRT for nasopharyngeal and oropharyngeal tumors and to assess the correlations with the functioning of the parotid glands.

\section{Patients and methods}

\section{Patient characteristics}

Between April 2009 and January 2010, six patients with nasopharyngeal tumors and four patients with oropharyngeal tumors were treated with IMRT at the Division of Radiotherapy, Hokkaido University Hospital. Written informed consent for IMRT was obtained from all patients. Histologically, one patient with tonsil lesions was diagnosed with non-Hodgkin's lymphoma, and six patients with nasopharyngeal tumors and three patients with oropharyngeal tumors were diagnosed with squamous cell carcinomas. The patients were six males and four females (mean age 64 years, age range 40-85 years). Clinically, the tumors were diagnosed as $\mathrm{T} 1$ in two patients, $\mathrm{T} 2$ in four, and $\mathrm{T} 3$ in four, and the nodal involvement was N0 in three patients, N1 in one, N2a in two, and N2b in four (Table 1). Seven of the ten patients received concurrent chemotherapy consisting of CDDP (cisplatin) (six patients) and TPF (taxotere + cisplatin + 5-fluorouracil) (one patient).

Parotid sizes and computed tomography (CT) numbers

The sizes and CT numbers of the bilateral parotid glands were determined on axial plain CT images before and after IMRT. Cross-sectional long/short diameters, showing the maximum area of the parotid glands, were also measured. The axial scan that showed the fewest artifacts caused by metal restorations and crowns in the oral cavity and had the largest size was selected to calculate the CT numbers of the parotid glands. The posterolateral regions of the parotid glands avoiding the retro-mandibular veins were selected to calculate the $\mathrm{CT}$ numbers. The $\mathrm{CT}$ numbers were determined three times, and the mean CT number was used as the representative $\mathrm{CT}$ number for a parotid gland (Fig. 1a, b).

Saxon test and visual analog scale (VAS)

As an objective assessment of salivation, we conducted the Saxon test before and after IMRT. The Saxon test was performed in the morning at our outpatient clinic. Patients were instructed not to drink, eat, or smoke for $2 \mathrm{~h}$ before the examination. In the Saxon test, saliva production was measured by weighing gauze before and after $2 \mathrm{~min}$ of chewing without swallowing. As a subjective assessment of salivation, we applied a VAS for xerostomia. The VAS was a horizontal $100-\mathrm{mm}$ line with vertical anchoring lines. The description used at the left end $(0 \mathrm{~mm})$ was "No feeling of dry mouth" and that at the right end $(100 \mathrm{~mm})$ was "Feeling dry mouth as severe as it can be". The CT examination, Saxon test, and VAS were performed within 1 week before the start of IMRT and after the completion of IMRT in each patient.

\section{Statistical analysis}

The changes in the bilateral parotid sizes and CT numbers before and after IMRT were compared with the Saxon test and VAS. Differences between the parotid glands on the ipsilateral side and the contralateral side were compared with a Student's $t$-test. The relationship between the parotid morphological and functional changes was evaluated with a Spearman rank correlation test. Statistical analyses were carried out using StatView Version 5 (SPSS, Cary, NC).
Table 1 Patient and tumor characteristics

$N P$ nasopharynx, $O P$ oropharynx, $p$ posterior, $u$ upper, $r t$ right, $l t$ left, $S C C$ squamous cell carcinoma, $M L$ malignant lymphoma; $T P F$ taxotere + cisplatin +5 fluorouracil, $C D D P$ cisplatin

\begin{tabular}{llllll}
\hline Patient no. & Age/sex & Primary site & TNM & Pathology & Chemotherapy \\
\hline 1 & 59/F & NP (p-lt wall) & T2N2bM0 & SCC & TPF \\
2 & $68 / \mathrm{M}$ & OP (rt tonsil) & T3N0M0 & SCC & CDDP \\
3 & $73 / \mathrm{M}$ & OP (rt tonsil) & T3N2bM0 & ML & \\
4 & $45 / \mathrm{F}$ & NP (rt wall) & T2N2M0 & SCC & CDDP \\
5 & $66 / \mathrm{F}$ & NP (rt wall) & T1N0M0 & SCC & CDDP \\
6 & $71 / \mathrm{F}$ & OP (rt tonsil) & T2N2bM0 & SCC & CDDP \\
7 & $40 / \mathrm{M}$ & NP (rt wall) & T3N1M0 & SCC & CDDP \\
8 & $77 / \mathrm{M}$ & NP (lt wall) & T1N2M0 & SCC & \\
9 & $56 / \mathrm{M}$ & OP (lt tonsil) & T2N2bM0 & SCC & CDDP \\
10 & 85/M & NP (u-p wall) & T3N0M0 & SCC & \\
\hline
\end{tabular}




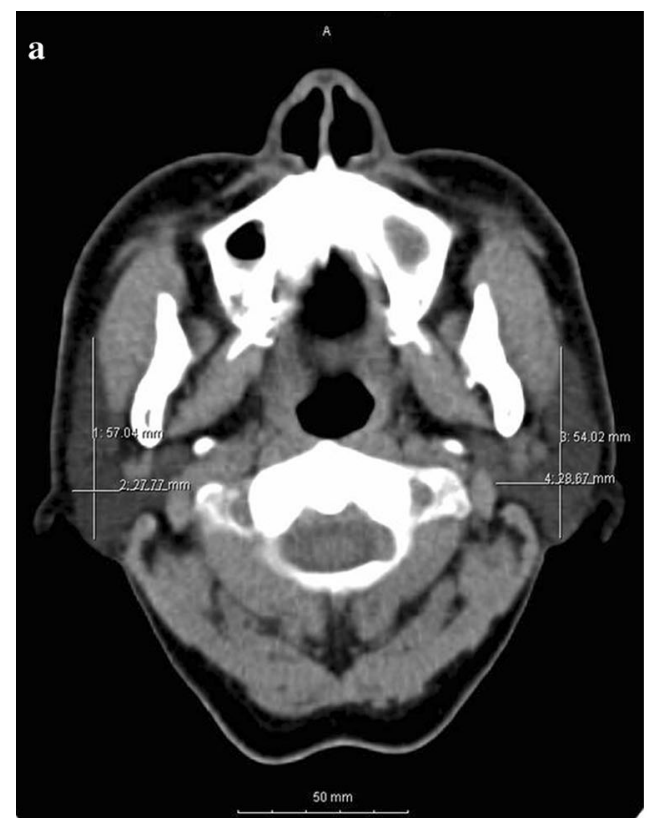

Fig. 1 a Method for determining the sizes of the parotid glands. Cross-sectional long/short diameters, showing the maximum area of the parotid glands, were measured on axial plain CT images.

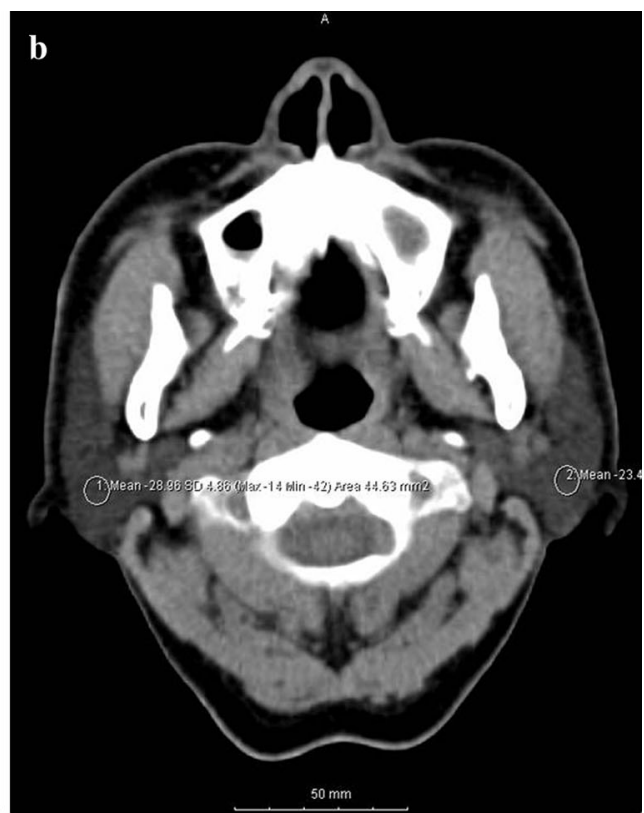

b Method for determining the CT numbers of the parotid glands. Circular regions of interest were selected on the posterolateral regions of the parotid glands, avoiding the retro-mandibular veins
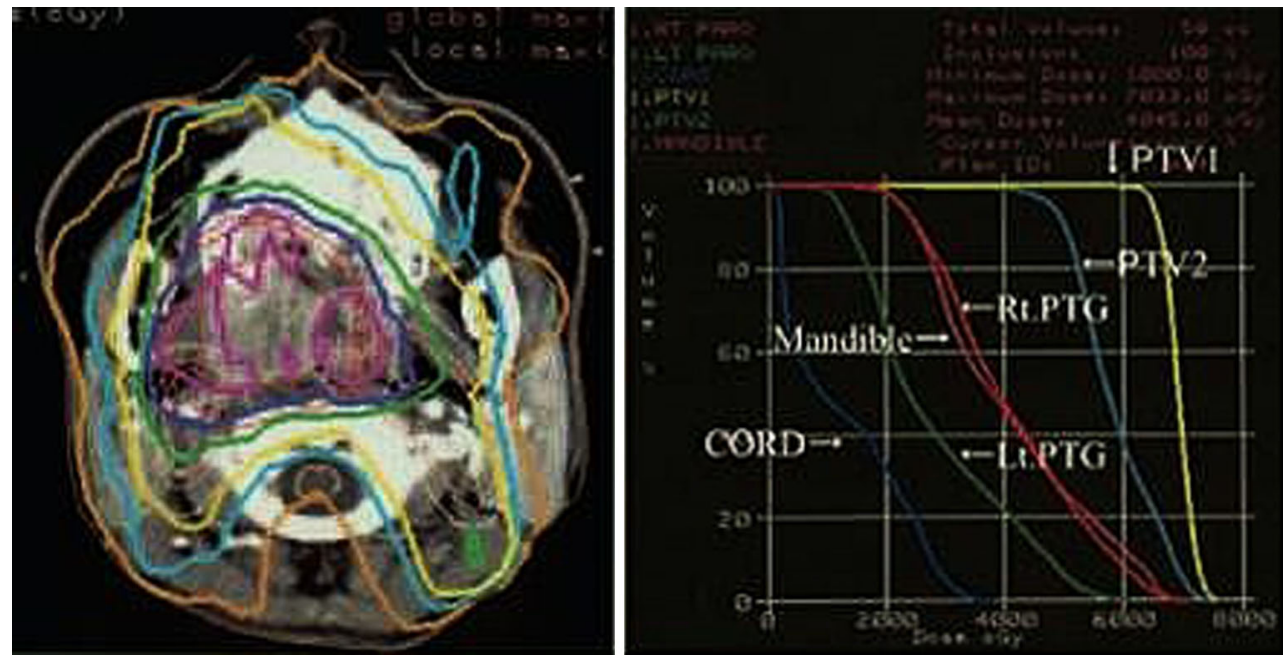

Fig. 2 Dose distribution and dose-volume histogram for a patient with an oropharyngeal tumor

Values of $p<0.05$ were considered to indicate statistical significance.

\section{Results}

The total dose to planning target volume (PTV) was 70 Gy in 35 fractions for squamous cell carcinomas and $50 \mathrm{~Gy}$ in 25 fractions for malignant lymphomas. The ipsilateral parotid glands were partially included in the PTV (Fig. 2). The mean dose was $41.5 \mathrm{~Gy}$ (33.9-68.4 Gy) for the ipsilateral parotid glands and 31.7 Gy (26.0-43.5 Gy) for the contralateral parotid glands. The average saliva secretion was $6.1 \mathrm{~g}$ (0.7-21.7 g) before IMRT and $1.4 \mathrm{~g}$ $(0.3-4.1 \mathrm{~g})$ after IMRT, giving a reduction rate of $69.5 \%$ $(0-90.0 \%)$. For the subjective assessment of xerostomia, the VAS varied depending on the patients, and the mean score was 6.6 (2-10) (Table 2). There was no correlation between the total dose to the bilateral parotid glands and the reduction in salivation $(r=0.26)$ (Fig. 3). There was a significant correlation between the reduction in salivation and the VAS $(r=0.64, p<0.05)$ (Fig. 4). The rate of 
Table 2 Doses to PTV and bilateral parotid glands, saliva secretion, and VAS

\begin{tabular}{|c|c|c|c|c|c|c|}
\hline Patients & $\begin{array}{l}\text { Total } \\
\text { dose } \\
\text { (Gy/ } \\
\text { fractions) }\end{array}$ & $\begin{array}{l}\text { Parotid } \\
\text { mean } \\
\text { dose } \\
\text { (Gy) } \\
\text { (ipsi/ } \\
\text { cont) }\end{array}$ & $\begin{array}{l}\text { Pre- } \\
\text { RT (g/ } \\
2 \text { min) }\end{array}$ & $\begin{array}{l}\text { Post- } \\
\text { RT (g/ } \\
2 \text { min) }\end{array}$ & $\begin{array}{l}\text { Reduction } \\
\text { rate }(\%)\end{array}$ & $\mathrm{V}$ \\
\hline 1 & $70 / 35$ & $39.1 / 28.3$ & 0.7 & 0.7 & 0 & 3 \\
\hline 2 & $70 / 35$ & $38.5 / 29.0$ & 7.5 & 4.1 & 45.3 & 6 \\
\hline $3 *$ & $50 / 25$ & $38.0 / 26.5$ & 4.4 & 0.6 & 86.4 & 8 \\
\hline 4 & $70 / 35$ & $58.8 / 43.5$ & 8.9 & 1.5 & 83.1 & 10 \\
\hline 5 & $70 / 35$ & $38.7 / 35.5$ & 5.0 & 0.5 & 90.0 & 9 \\
\hline $6 \#$ & $70 / 35$ & $68.4 / 32.1$ & 4.6 & 1.1 & 76.1 & 3 \\
\hline 7 & $70 / 35$ & $37.6 / 33.5$ & 4.1 & 0.6 & 85.2 & 9 \\
\hline 8 & $70 / 35$ & $33.9 / 33.5$ & 3.7 & 0.5 & 86.5 & 5 \\
\hline 9 & $70 / 35$ & $36.4 / 29.3$ & 21.7 & 3.9 & 82.3 & 7 \\
\hline 10 & $70 / 35$ & $26.0 / 25.9$ & 0.9 & 0.3 & 60.0 & 6 \\
\hline Mean & & $41.5 / 31.7$ & 6.1 & 1.4 & 69.5 & 6.6 \\
\hline
\end{tabular}

ipsi Ipsilateral, cont contralateral, $R T$ radiotherapy, $V A S$ visual analog scale

* A case of malignant lymphoma

\# Lymph node metastasis in the ipsilateral parotid gland

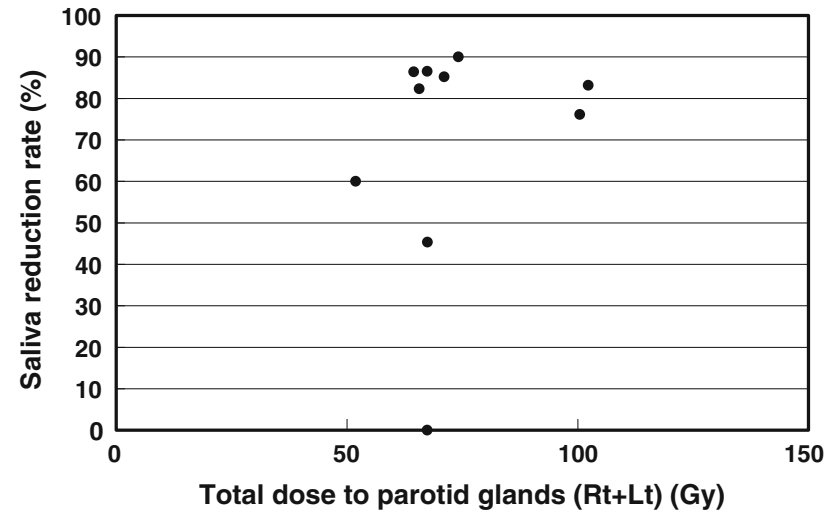

Fig. 3 Relationship between the total dose to the bilateral parotid glands and the reduction in salivation. The Spearman rank correlation coefficient is 0.26

reduction in the parotid gland size was $39.4 \%$ $(23.8-50.7 \%)$ for the ipsilateral side and $26.5 \%$ (12.9-44.9) for the contralateral side, and the difference was significant $(p<0.02)$ (Fig. 5). No significant correlation was established between the bilateral reduction in the parotid size and salivation $(r=0.32)$ (Fig. 6). The CT number was reduced by $13.8(-28.8$ to +17.0$)$ for the ipsilateral side and $5.4(-14.8$ to +14.3$)$ for the contralateral side, and the difference was significant $(p<0.05)$ (Fig. 7). There was a significant strong correlation between the reductions in the CT number and salivation $(r=0.84$, $p<0.01$ ) (Fig. 8). It was observed that the CT numbers of

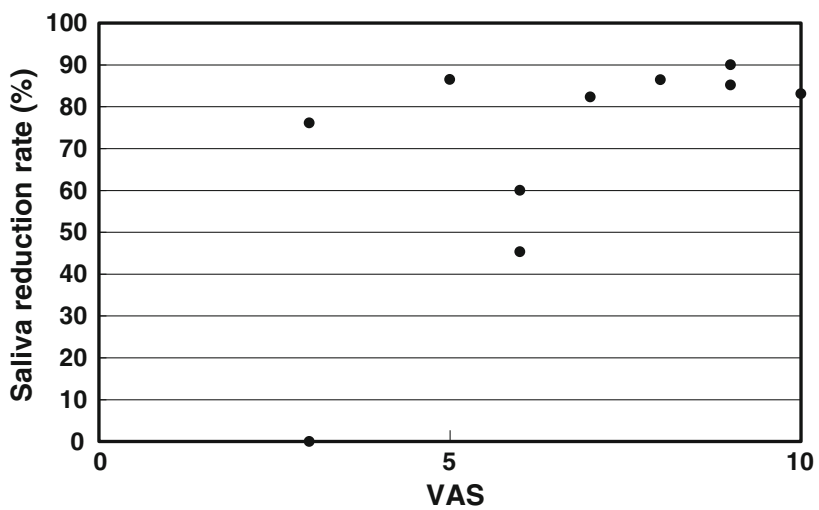

Fig. 4 Relationship between the reduction in salivation and the VAS The Spearman rank correlation coefficient is $0.64(p<0.05)$
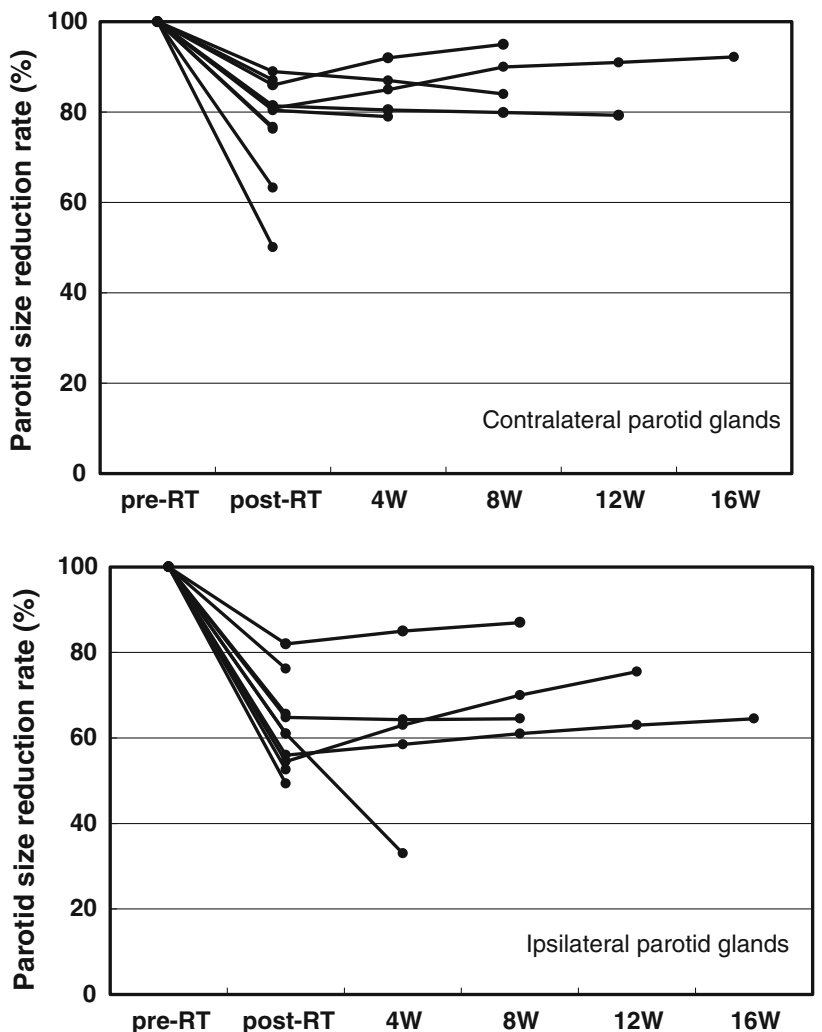

Fig. 5 Comparison of the rate of the size reduction between the ipsilateral and contralateral parotid glands. The difference is significant $(p<0.02$, Student's $t$-test). $R T$ radiotherapy, $W$ week

the parotid glands tend to revert after completion of IMRT (Figs. 5, 7).

\section{Discussion}

IMRT is a newly introduced technique that aims to spare radiosensitive organs while delivering adequate doses to tumors. Initially, prostate cancer was treated with IMRT to 


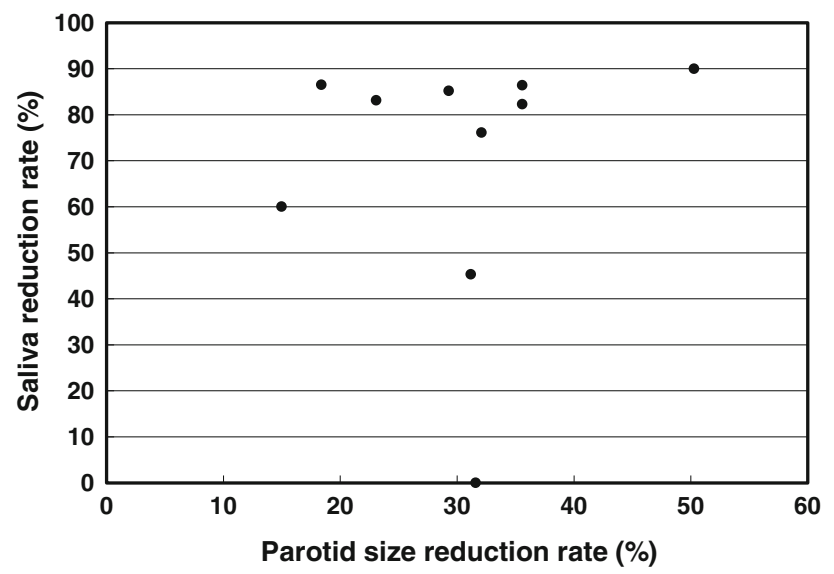

Fig. 6 Relationship between the reduction in the parotid size and salivation. The Spearman rank correlation coefficient is 0.32
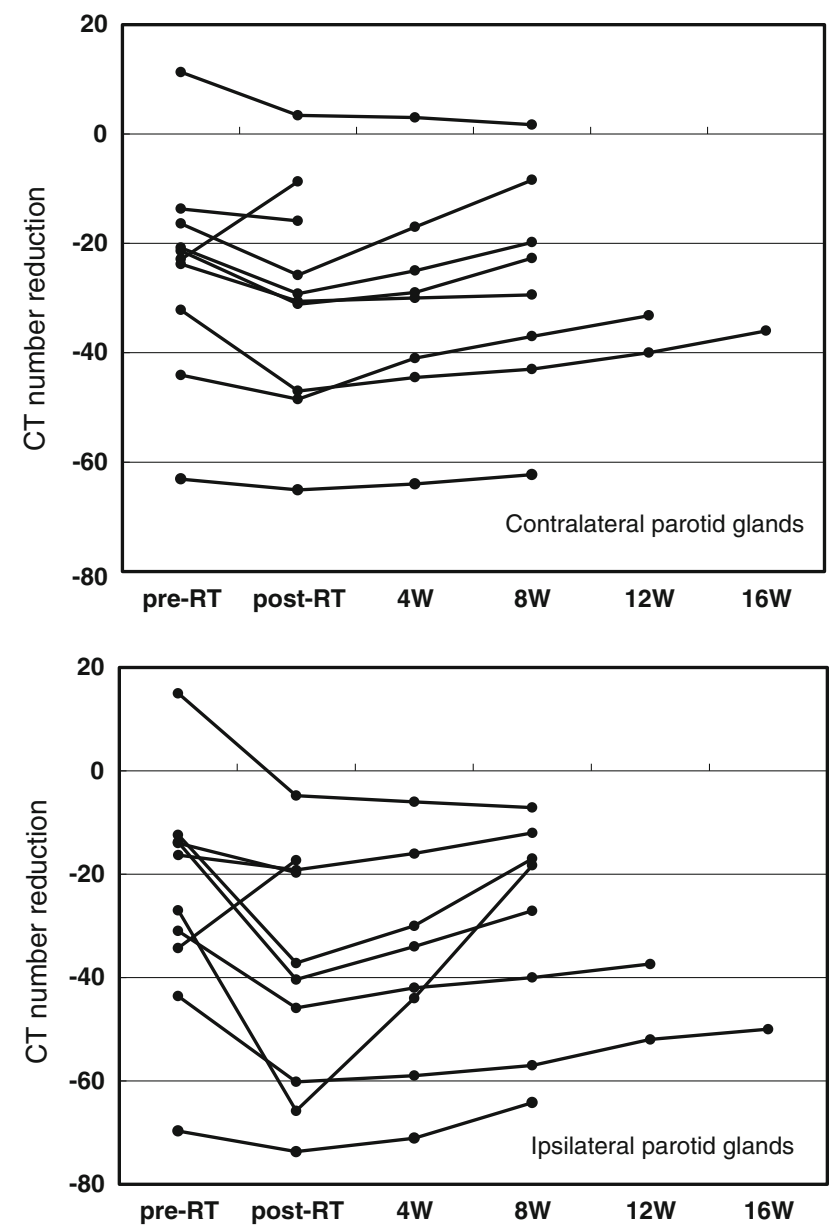

Fig. 7 Comparison of the reduction in the CT numbers between the ipsilateral and contralateral parotid glands. The difference is significant ( $p<0.05$, Student's $t$-test). $R T$ radiotherapy, $W$ week

prevent adverse effects on the rectum [5]. Many patients treated with radiotherapy for head and neck malignancies suffer from xerostomia, which causes reductions of patient

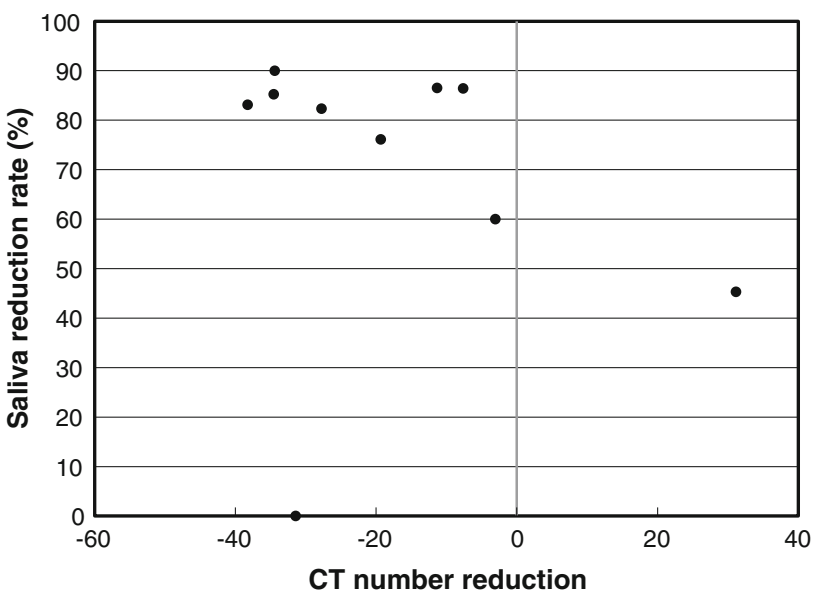

Fig. 8 Relationship between the reduction in the CT numbers and salivation. The Spearman rank correlation coefficient is 0.84 $(p<0.01)$

quality of life, including difficulty of speaking and swallowing, as well as taste loss and oral infections [2]. There have been several attempts to reduce radiation damage to the major salivary glands in radiotherapy $[3,4]$. In this study, IMRT was applied to preserve the functions of the salivary glands. In most IMRT studies, much effort goes into reducing the parotid gland dose. A number of papers have reported theoretical values for acceptable IMRT doses in the reduction of parotid gland doses [6]. However, only a few reports have dealt with measurements to assess the effects of reducing the gland doses. Marks et al. [7] observed a marked reduction in salivary flows in glands receiving more than 30-40 Gy. Leslie and Dische [8] found that the average parotid salivary flow was 60-70\% of the baseline following 10-14 Gy and undetectable in glands receiving 40-42 Gy [8]. They concluded that the dose required for an optimum effect lies between 15 and $40 \mathrm{~Gy}$. In the present study, we used the Saxon test to measure the saliva output, because it reflects the function of the parotid glands as stimulated salivation rather than unstimulated salivation, which is predominantly from the submandibular glands. The mean dose to the parotid gland for the ipsilateral and contralateral parotid glands was $41.5 \mathrm{~Gy}$ and $31.7 \mathrm{~Gy}$, with size reductions of 39.4 and $26.5 \%$, respectively $(p<0.02)$. Although the saliva flow from each parotid gland was not separately determined, this result may still reflect a dose-dependent morbidity of the parotid gland function, and the threshold beyond which damage becomes prominent may lie around $30 \mathrm{~Gy}$. The saliva reduction rate in this study was $69.5 \%$, which is comparable to other studies. Although feelings of mouth dryness are very subjective and affected by variability, the present measurements showed significant correlations between the objective saliva output and the Saxon test and VAS. These results indicate that the VAS, reflecting the 
subjective patient sensation of mouth dryness, is a useful and concise parameter to assess xerostomia after radiotherapy. Teshima et al. [9] evaluated the correlation between parotid volume and saliva production in patients treated with preoperative radiotherapy for oral cancers. They reported that after 30 Gy of irradiation, the mean saliva production decreased from 4.2 to $1.0 \mathrm{~g}$ and the postradiotherapy/pre-radiotherapy parotid volume ratio ranged from 54 to $85 \%$ (mean $71 \%$ ) [9]. They also indicated that the post-radiotherapy/pre-radiotherapy parotid volume ratio was inversely and significantly correlated with the amount of post-radiotherapy saliva reduction [9]. The reduction in the parotid gland size in the present study was $39.4 \%$ for the ipsilateral side and $26.5 \%$ for the contralateral side. These findings may demonstrate that IMRT achieved the aim of alleviating the adverse effects on the contralateral parotid glands, taking into consideration the fact that almost all patients in this study were administered 70 Gy of irradiation and that the difficulties in IMRT planning because of the reduced laterality resulted from the tumor staging and occurrence site. Although there was no significant correlation between the reduction in the parotid gland sizes and saliva production, this is likely to arise from the small sample size in this study. There have been no other reports of CT numbers of salivary glands after radiotherapy. Heo et al. [10] reported a quantitative analysis of normal major salivary glands using CT, and reported that the CT numbers varied widely with sex, age, and obesity state (body mass index). Similar to the previous reports, the $\mathrm{CT}$ numbers of the patients in the present study varied from -69.7 to +15.0 . A reduction in the $\mathrm{CT}$ number was observed in 9 of the 10 patients in this study, and the range of the reduction was significantly larger in the ipsilateral parotid gland than in the contralateral parotid gland $(p<0.05)$. Numerous investigators have described the nature of salivary gland damage after irradiation in animal studies [10-13]. The irradiated parotid glands are characterized by significant fibrosis, acinar atrophy, and parenchymal loss $[10,11]$. It is known that radiation-induced fatty replacement of bone marrow is the most common osseous abnormality observed in patients with doses as low as 800 cGy [14]. Furthermore, Tartaglino et al. [15] reported that muscle and salivary gland atrophy with fatty replacement may arise from radiation-induced changes in the head and neck. Sagowski et al. [13] reported that progressive vacuolization (30 Gy) developed into lipomatosis (46 Gy) in rat parotid glands. The reductions in the CT numbers after radiotherapy in the present study may have resulted from acinar atrophy, parenchymal loss, and fatty replacement, which would be in agreement with the previous reports. On the contrary, another report demonstrated increased attenuation and enhancement as well as atrophy of salivary glands after radiotherapy evaluated by contrast- enhanced CT [14]. The enhancements of the salivary glands may be caused by contrast material leakage owing to increased vascular permeability or an increase in the extracellular space owing to a diminished number of acini [14]. Although the CT scans obtained in the present study were plain (non-enhanced), one patient showed an increase in the CT number. This patient complained of parotid tenderness after the radiotherapy, and may have been affected by sialadenitis. This could be a result of increases in water content caused by edema of the inflamed parotid gland. Nomayr et al. [16] reported that after irradiation of 70 Gy, salivary gland edema, corresponding to sialadenitis, was detected in 2 of 9 cases (parotid gland) and 4 of 13 cases (submandibular gland). The present study may provide additional insight into the effects of radiation-induced salivary gland injury. Among the morphological changes of the parotid glands, the CT number can also be a predictor of the function of saliva production after radiotherapy. Further studies of this matter are necessary, including attention to the morphological changes of submandibular/ sublingual glands with longer follow-up periods and comparisons of IMRT with conventional RT.

Acknowledgments This study was partially supported by a Grant from Amino-Up Chemical Co. Ltd.

Conflict of interest Kenichi Obinata, Motoyasu Nakamura, Marco Carrozzo, Iain Macleod, Andrew Carr, Shinichi Shirai, and Hitoshi Ito declare that they have no conflict of interest.

Open Access This article is distributed under the terms of the Creative Commons Attribution License which permits any use, distribution, and reproduction in any medium, provided the original author(s) and the source are credited.

\section{References}

1. Ghadjar P, Simcock M, Zimmermann F, Betz M, Bodis S, Bernier J, et al. Predictors of severe late radiotherapy-related toxicity after hyperfractionated radiotherapy with or without concomitant cisplatin in locally advanced head and neck cancer. Secondary retrospective analysis of a randomized phase III trial (SAKK 10/94). Radiother Oncol. 2012;104:213-8.

2. Deboni AL, Giordani AJ, Lopes NNF, Dias RS, Segreto RA, Jensen SB, et al. Long-term oral effects in patients treated with radiochemotherapy for head and neck cancer. Support Care Cancer. 2012;20:2903-11.

3. Eisbruch A, Haken RKT, Kim HM, Marsh LH, Ship JA. Dose, volume, and function relationships in parotid salivary glands following conformal and intensity-modulated irradiation of head and neck cancer. Int J Radiat Oncol Biol Phys. 1999;45:577-87.

4. Voordecker M, Farrag A, Everaert H, Tournel K, Storme G, Verellen D, et al. Parotid gland sparing with helical tomotherapy in head and neck cancers. Int J Radiat Oncol Biol Phys. 2012;84:443-8.

5. Meerleer GOD, Vakaet LAML, Gersem WRTD, Wagter CD, Naeyer BD, Neve WD. Radiotherapy of prostate cancer with or 
without intensity modulated beams: a planning comparison. Int J Radiat Oncol Biol Phys. 2000;47:639-48.

6. Deasy JO, Moiseenko V, Marks L, Chao KS, Nam J, Eisbruch A. Radiotherapy dose-volume effects on salivary gland function. Int J Radiat Oncol Biol Phys. 2010;76:58-63.

7. Marks JE, Davis CC, Gottsman VL, Purdy JE, Lee F. The effects of radiation on parotid salivary function. Int J Radiat Oncol Biol Phys. 1981;7:1013-9.

8. Leslie MD, Dische S. The early changes in salivary gland function during and after radiotherapy given for head and neck cancer. Radiother Oncol. 1994;30:26-32.

9. Teshima K, Murakami R, Tomitaka E, Nomura T, Toya R, Hiraki A, et al. Radiation-induced parotid gland changes in oral cancer patients: correlation between parotid volume and saliva production. Jpn J Clin Oncol. 2010;40:42-6.

10. Heo MK, Lee SC, Lee SS, Choi HM, Choi SC, Park TW. Quantitative analysis of normal major salivary glands using computed tomography. Oral Surg Oral Med Oral Pathol Oral Radiol Endod. 2001;92:240-4.

11. Stephens LC, King GK, Peters LJ, Kian K, Schultheiss TE, Jardine JH. Acute and late injury in rhesus monkey parotid glands. Am J Pathol. 1986;124:469-78.
12. Radfar L, Sirois DA. Structural and functional injury in minipig salivary glands following fractionated exposure to $70 \mathrm{~Gy}$ of ionizing radiation: an animal model for human radiation-induced salivary gland injury. Oral Surg Oral Med Oral Pathol Oral Radiol. 2003;96:267-74.

13. Sagowski C, Wenzel S, Tesche S, Jenicke L, Jaehne M. Investigation of radiosialadenitis during fractioned irradiation: sialoscintigraphical and histomorphological findings in rats. Eur Arch Otorhinolaryngol. 2003;260:513-7.

14. Rabin BM, Meyer JR, Berlin JW, Marymount MH, Palka PS, Russell EJ. Radiation-induced changes in the central nervous system and head and neck. Radiographics. 1996;16:1055-72.

15. Tartaglino LM, Rao VM, Markiewicz DA. Imaging of radiation changes in the head and neck. Semin Roentgenol. 1994;29:81-91.

16. Nomayr A, Lell M, Sweeney R, Bautz W, Lukas P. MRI appearance of radiation induced changes of normal cervical tissues. Eur Radiol. 2001;11:1807-17. 